BNL-112690-2016-JA

File \# 93622

\title{
Terasonic Excitations in 2D Gold Nanoparticle Arrays in a Water Matrix as Revealed by Atomistic Simulations
}

\author{
Dima Bolmatov, Mikhail Zhernenkov, \\ Dmitry Zav'yalov, Yong Q. Cai, and Alessandro Cunsolo
}

Submitted to Journal of Physical Chemistry C

Aug 19, 2016

Photon Sciences Department

Brookhaven National Laboratory

\author{
U.S. Department of Energy \\ USDOE Office of Science (SC), \\ Basic Energy Sciences (BES) (SC-22)
}

Notice: This manuscript has been authored by employees of Brookhaven Science Associates, LLC under Contract No. DE- SC0012704 with the U.S. Department of Energy. The publisher by accepting the manuscript for publication acknowledges that the United States Government retains a non-exclusive, paid-up, irrevocable, world-wide license to publish or reproduce the published form of this manuscript, or allow others to do so, for United States Government purposes. 


\section{DISCLAIMER}

This report was prepared as an account of work sponsored by an agency of the United States Government. Neither the United States Government nor any agency thereof, nor any of their employees, nor any of their contractors, subcontractors, or their employees, makes any warranty, express or implied, or assumes any legal liability or responsibility for the accuracy, completeness, or any third party's use or the results of such use of any information, apparatus, product, or process disclosed, or represents that its use would not infringe privately owned rights. Reference herein to any specific commercial product, process, or service by trade name, trademark, manufacturer, or otherwise, does not necessarily constitute or imply its endorsement, recommendation, or favoring by the United States Government or any agency thereof or its contractors or subcontractors. The views and opinions of authors expressed herein do not necessarily state or reflect those of the United States Government or any agency thereof. 


\section{Terasonic Excitations in 2D Gold Nanoparticle}

\section{Arrays in a Water Matrix as Revealed by Atomistic Simulations}

Dima Bolmatov, ${ }^{* \dagger}$ Mikhail Zhernenkov, $^{\dagger}$ Dmitry Zav'yalov, ${ }^{\ddagger}$ Yong Q. Cai, ${ }^{\dagger}$ and Alessandro Cunsolo ${ }^{\dagger}$

National Synchrotron Light Source II, Brookhaven National Laboratory, Upton, NY 11973, USA, and Volgograd State Technical University, Volgograd, 400005 Russia

E-mail: d.bolmatov@gmail.com

${ }^{*}$ To whom correspondence should be addressed

${ }^{\dagger}$ National Synchrotron Light Source II, Brookhaven National Laboratory, Upton, NY 11973, USA

†Volgograd State Technical University, Volgograd, 400005 Russia 


\begin{abstract}
In this work we report on terahertz phononic excitations in 2D gold nanoparticle arrays in a water matrix through a series of large-scale molecular dynamics simulations. For the first time, we observe acoustic Dirac-like crossings in $\mathrm{H}\left(\mathrm{H}_{2} \mathrm{O}\right)$ atomic (molecular) networks which emerge due to an intraband phononic scattering. These crossings are the phononic fingerprints of ice-like arrangements of $\mathrm{H}\left(\mathrm{H}_{2} \mathrm{O}\right)$ atomic (molecular) networks at nanometer scale. We reveal how phononic excitations in metallic nanoparticles and the water matrix reciprocally impact on one another providing the mechanism for the $\mathrm{THz}$ phononics manipulation via structural engineering. Furthermore, we show that by tuning the arrangement of $2 \mathrm{D}$ gold nanoparticle assemblies the Au phononic polarizations experience sub-terahertz hybridization (Kohn anomaly) due to surface electron-phonon relaxation processes. This opens the way for the sound control and manipulation in soft matter metamaterials at nanoscale.
\end{abstract}

A new frontier of nanotechnology is the growth of complex nanoparticles arrays selfassembling into defect-free crystallites arranged with nanometer periodicity, potentially offering unexplored functionalities. ${ }^{1}$ For instance, the manipulation of their acoustic properties provides the opportunity to minimize thermal losses in modern applications. ${ }^{2,3}$ Ideal candidates to implement the heat flow control based on structural engineering are phononic crystals, ${ }^{4-6}$ i.e. composite materials whose structural arrangement deeply interferes with acoustic waves of certain frequencies, reflecting, trapping, slowing down or even stopping them. ${ }^{7-9}$ These metamaterials have been demonstrated to be useful in a variety of applications, such as optical cooling, energy harvesting and management of thermal conductivity. ${ }^{1}$ Although the search for new classes of phononic crystals is rapidly maturing, the development of devices operating at extremely high (terahertz) frequencies still remains essentially unexplored. Such a drawback is particularly penalizing since the heat transport utilizes terahertz ( $\mathrm{THz}$ ) phonons as carriers. In order to produce phononic crystals efficient in $\mathrm{THz}$ frequency window, nanoparticle (NP) assemblies must be arranged in superlattices with inter-particle distances comparable with the nanometer-wavelengths of $\mathrm{THz}$ phonons. 
Phonons, classically known as elastic waves, are bosons and in solids have three independent polarizations: one is longitudinal (a compression wave) and two of these are transverse (shear waves). It has recently been analytically demonstrated that those materials, which lack long-range order pair correlations exhibit low-frequency transverse phononic gaps in the vibrational spectrum. ${ }^{10}$ For instance, new experimental studies provided a compelling evidence for the existence of the low-frequency shear phononic gaps ${ }^{11,12}$ in liquids, which is a result of symmetry breaking ${ }^{13}$ due to the interaction of phonons. ${ }^{10,13}$ It was revealed that liquids undergo a viscous-to-elastic crossover at sub-terahertz frequency scale (terasonic excitations). ${ }^{12}$ This implies that at elevated frequencies soft matter systems exhibit a solid-like dynamic response, ${ }^{14,15}$ thus, they are capable of supporting the propagation of longitudinal as well as transverse sub- $\mathrm{THz}$ phononic modes similarly to isotropically disordered solids. ${ }^{11,12,14,16}$ Therefore, it may become plausible to advance the field of soft matter metamaterials at $\mathrm{THz}$ frequency scale. Potentially relevant candidates may encompass programmable nano-architectures ${ }^{17,18}$ organized via DNA-mediated interactions ${ }^{19,20}$ with a particular emphasis on NP self-assemblies through shape-induced directional binding. ${ }^{21}$

In this article we report on the terasonic excitations in 2D (planar) gold NP arrays in a water matrix (see Fig.1) through a series of large-scale molecular dynamics (MD) simulations. The obtained results were explained in the framework of a unified phonon-based approach ${ }^{10}$ in which phononic excitations, dynamic and pair correlation functions are reconciled. ${ }^{11} \mathrm{We}$ show that phononic excitations of $2 \mathrm{D}$ gold NP arrays and the water matrix have reciprocal impact on one another, thus, it can be tuned via structural engineering. The interactions among all ingredients of the hybrid system (see Fig.1) are defined in the Methods section.

Phononics in $2 D$ gold NP arrays in a water matrix. Dirac dispersion relations, initially introduced in relativistic particle physics, have recently attracted much interest due to the suitable manufacture of a monolayer of hexagonally arranged carbon atoms known as a graphene sheet. The unique energy dispersion of electronic and phononic band structure leads to astonishing phenomena including, but not limited to electromagnetic waves ${ }^{24}$ and 


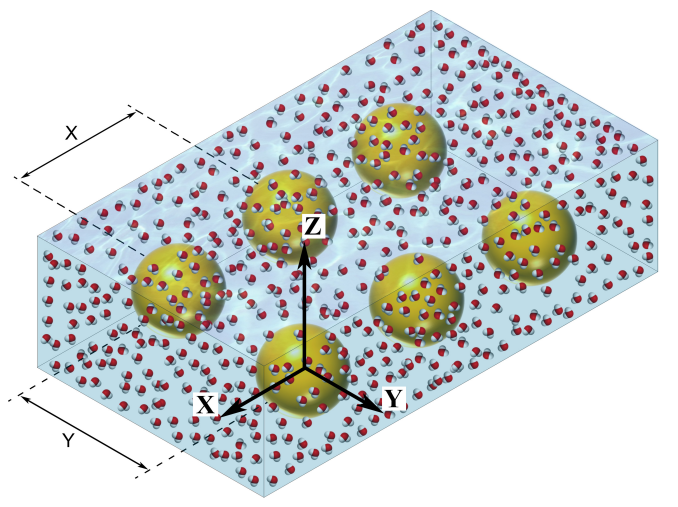

Figure 1: Schematic of the 2D gold NP array in a water matrix.

sound waves in 2D materials, ${ }^{25}$ one-way edge phononic modes with topologically driven band gaps, ${ }^{26}$ Zitterbewegung oscillations, ${ }^{27}$ plasmonic Dirac cones in metallic NPs,${ }^{28}$ topological boundary modes in isostatic lattices, ${ }^{29}$ chiral phonons at high symmetry points in hexagonal lattices, ${ }^{30}$ elastic waves and helical edge states in phononic topological insulators. ${ }^{31,32}$ In all these phenomena, it is evident that the arrangement and the geometry of artificially assembled materials strongly impact the wave propagation regardless of the nature of waves, electromagnetic or elastic, and the scale in which they operate. Below we prove this notion by considering a soft matter system of 2D gold NP array (see Fig.1) with two different geometries. The choice of Au NPs in our study is justified by an extensive use of them in modern ultrasound therapeutic applications ${ }^{22}$ primarily being relevant to the treatment of cancer. $^{23}$

Lattice and finite size effects. It is well-known that sound waves, both compression and shear, may propagate in structurally-ordered bulk solids over longer distances as compared to disordered materials. The impact of size reduction of NP assemblies strongly influences their phononic states, and prevents sound waves from propagating infinitely. Furthermore, the reduced size prevents low-frequency phononic states (long-wavelength phonons) from being excited. For example, 1-4 nm-sized Au clusters exhibit sub-terahertz acoustic gaps, which have been observed through vibrational density of states calculations. ${ }^{33}$ Nevertheless, the attempts to manipulate sound propagation via structural engineering in nm-sized NP 
assemblies confined in a soft matter matrix still remain elusive.

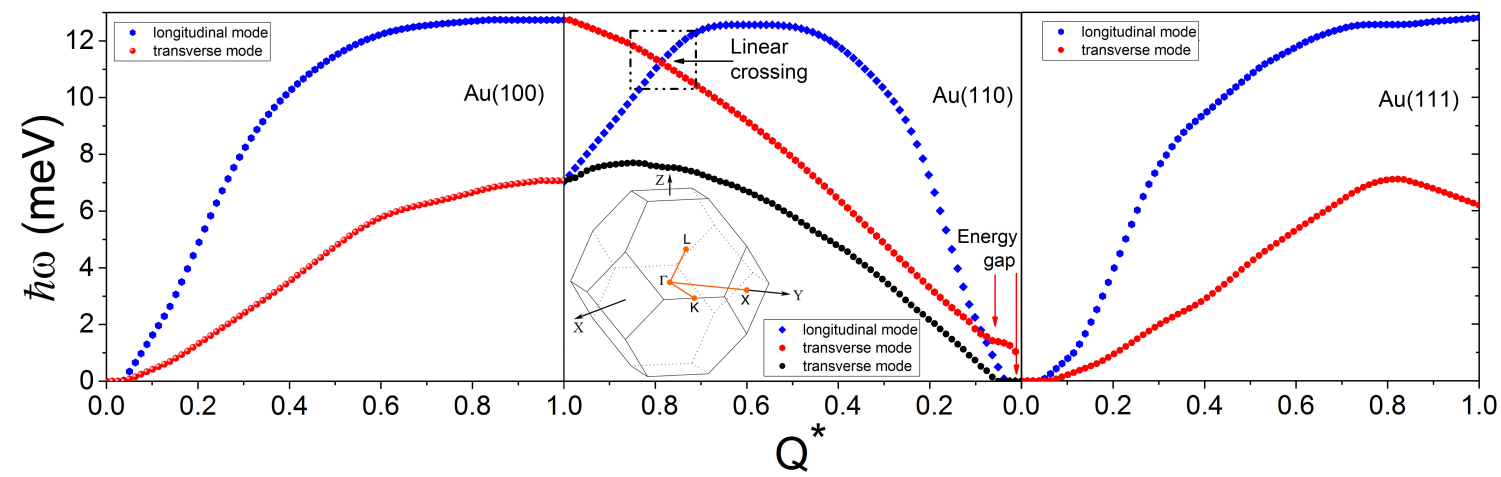

Figure 2: Phononic dispersion relations of a gold NP in 2D array (geometry I) along principal symmetry $\Gamma \mathrm{X}, \Gamma \mathrm{K}$ and $\Gamma \mathrm{L}$ directions calculated from MD simulations. $\mathrm{Q}^{*}$ is the reduced wavenumber. Emergence of low-frequency phononic gaps is the fingerprint of the finite size effect in NP assemblies.

In Fig.2 we show the Q-dispersions of terasonic excitations of $4.0 \mathrm{~nm}$ gold NP in 2D array along the principal $\Gamma \mathrm{X}, \Gamma \mathrm{K}$ and $\Gamma \mathrm{L}$ directions in geometry $I(\mathrm{x}=\mathrm{y}=5.0 \mathrm{~nm}$, see Fig.1), which closely resemble their bulk counterparts. ${ }^{34}$ In this geometry (NPs are tightly packed), we observe low-Q phononic gaps along all high symmetry directions. These are Q-regions where the propagation of the corresponding mode is forbidden, as revealed by a vanishing acoustic frequency. Due to the close proximity of spherical NPs' surfaces, which are $1.0 \mathrm{~nm}$ apart only, energy gaps are smoothed exhibiting an incipient behavior of the Kohn anomaly. ${ }^{35}$ Nevertheless, one energy gap is clearly visible along the $\mathrm{Au}(110)$ direction in the transverse phononic branch. The transverse phononic branch along the $\mathrm{Au}(110)$ direction exhibits a linear crossing with the longitudinal mode at high frequency regime mimicking a so-called Dirac-like point. The origin of a linear-crossing phononic point along $\mathrm{Au}(110)$ direction is not coincidental and can be understood as a confined water-induced effect triggered through resonant multiple scattering. The latter effect is captured by modeling Au arrays and the water matrix interactions with a standard potential. ${ }^{36}$ It was shown that a very simple modeling based on Lennard-Jones (LJ) interactions reproduces the essential features of various experiments providing a physically justified choice of $\mathrm{p}$ arameters ${ }^{36} \mathrm{~T}$ he fi nite di stance at which the inter-particle $(\mathrm{Au}-\mathrm{O})$ potential vanishes is equal to $\sigma_{A u O}=0.36 \mathrm{~nm},{ }^{36}$ see Meth- 

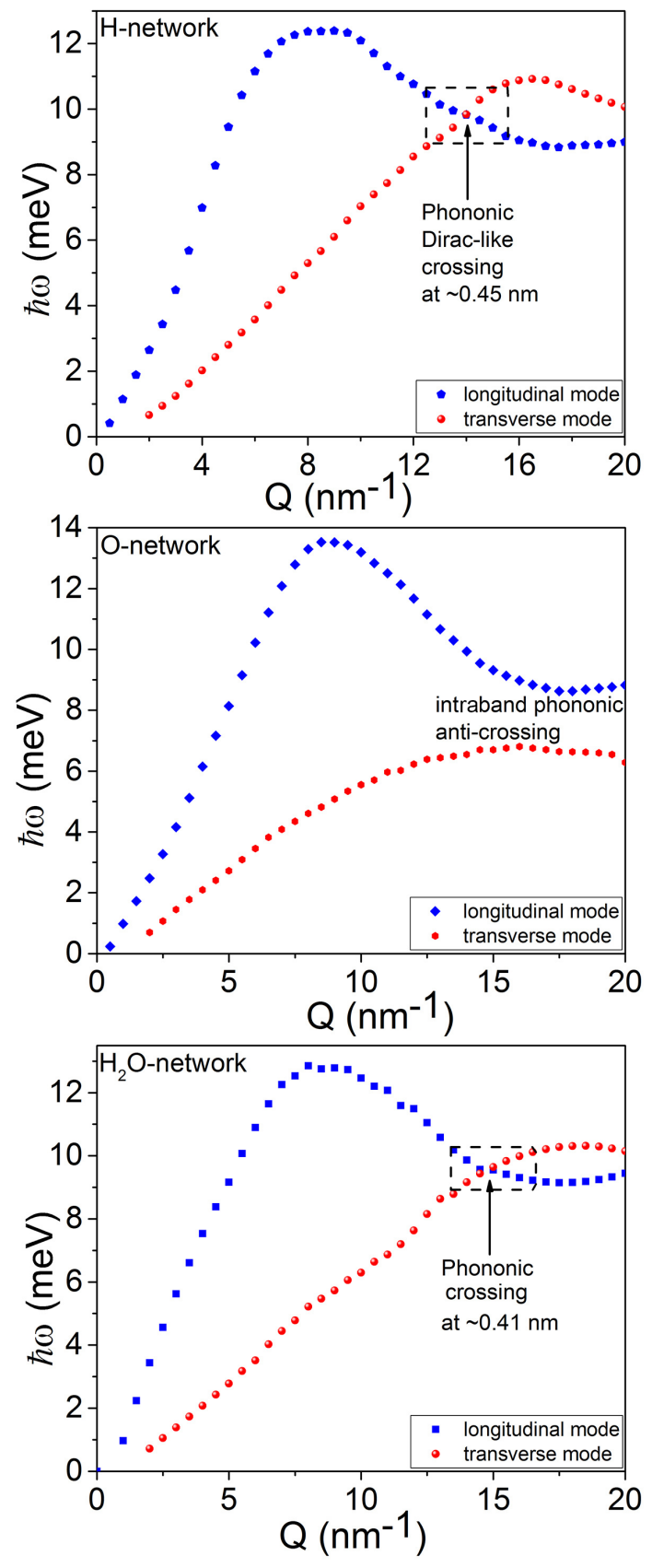

Figure 3: The dispersion curves of phonon excitations propagating through atomic (H,O) and molecular $\left(\mathrm{H}_{2} \mathrm{O}\right)$ networks as calculated for the geometry $I$. 
ods section for detail. Remarkably, a linear intraband crossing along $\mathrm{Au}(110)$ direction (see Fig.2) occurs at $\mathrm{Q}^{*} \sim 0.79$, where the reduced wavenumber was defined as $\mathrm{Q}^{*}=\frac{Q}{d}$ and $d=22$ $\mathrm{nm}^{-1}$ is the distance between $\Gamma$ and $\mathrm{K}$ high symmetry points along (110) direction. Therefore, this linear crossing occurs at $\mathrm{Q} \sim 17.4 \mathrm{~nm}^{-1}$ in the reciprocal space or, equivalently, at characteristic distance $d_{r e s}=\frac{2 \pi}{Q}=0.36 \mathrm{~nm}$ in the real space. Interestingly, $\sigma_{A u O}=d_{\text {res }}=0.36$ $\mathrm{nm}$ is the resonant distance at which the water-induced ( $\mathrm{Au}$ pair correlations at $d_{\text {res }}=0.36$ nm $g(0.36)_{A u A u} \sim 0$, see Fig.7) multiple phononic scattering takes place (see Fig.2).

In its liquid state, water molecules stick to one another through hydrogen bonding, constantly forming and breaking bonds as they rattle around. ${ }^{37}$ They can rearrange themselves not just one at a time, but in pairs as well. Two molecules can simultaneously break their hydrogen bonds with their neighbors and rotate about one another like gears. ${ }^{38}$ The reconfiguration o ccur via a quantum t unneling e ffect. ${ }^{38}$ This effect c an b e significantly enhanced when water molecules are confined to nanocavities in g emstones ${ }^{39}$ such as b eryl. ${ }^{40}$ The crystal structure of beryl contains channels with hexagonal cross-sections that can confine water molecules. The channels periodically narrow into cages roughly $0.5 \mathrm{~nm}$ wide by $0.9 \mathrm{~nm}$ long, and only big enough for one water molecule. ${ }^{40}$ Hence, water molecules may form hexagonallike structures ${ }^{41}$ at the $\mathrm{THz}$-frequency scale in nm-sized confined geometries. ${ }^{39,40}$ We recall, finally, that the hexagonal arrangements of hydrogen atoms characterize ice (Ih) structure, which is the most common form of ice in nature.

Here, we observe a dynamic fingerprint of an ice-like morphology in the water matrix via calculations of phononic dispersions (see Fig.3). We demonstrate how sound propagates over H-network, O-network, and $\mathrm{H}_{2} \mathrm{O}$-based network, the latter being primarily formed by covalent bonds. We find that phonon propagation in the water matrix is governed by H-network exhibiting a linear phononic intraband crossing at elevated frequencies, whereas phononic branches in the O-network avoid intraband mixing. It is well-known that fluid systems as liquid water are isotropically disordered materials. Thus, the obtained phononic dispersions were calculated by averaging over the course of a simulation run. They exhibit 
degenerate phononic states (two-fold degeneracy ${ }^{25}$ ) along the transverse polarizations (for instance, see Fig.3) revealing their Dirac-like ${ }^{31}$ nature along the edge/boundary of nanostructured pair correlated domains. Phononic Dirac-like states in H-network are shown to be robust against scattering from heavy oxygen atoms in O-network as well as $\mathrm{H}_{2} \mathrm{O}$-network in the water matrix continuum, which is as also evidenced via the Green-Kubo formalism below.

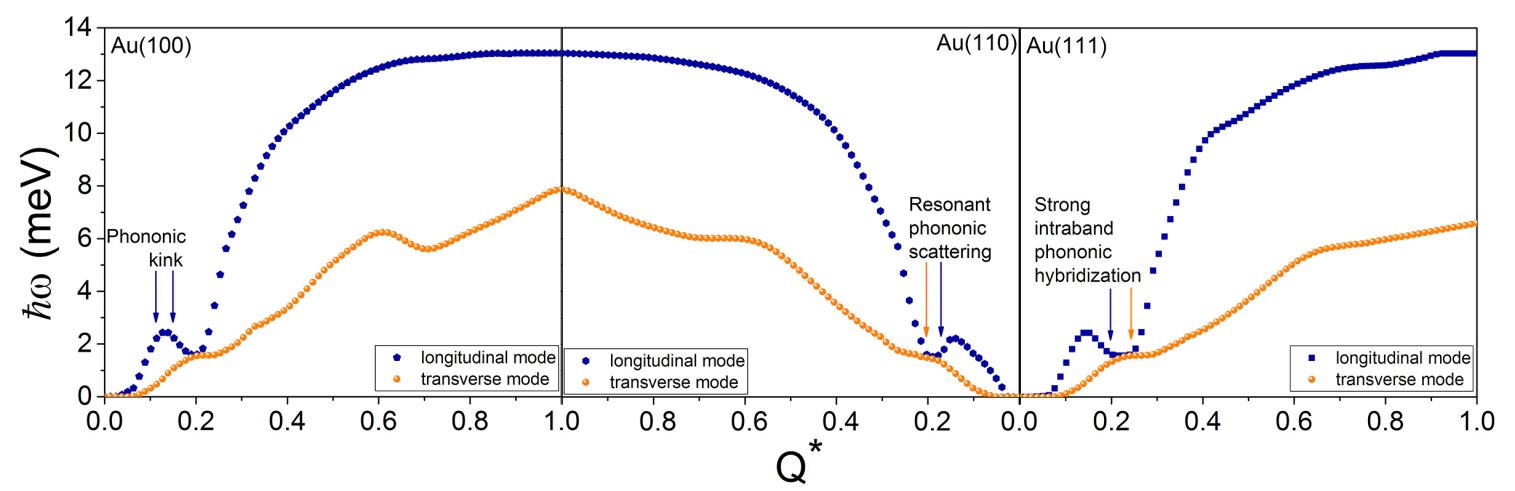

Figure 4: Phononic dispersion relations of a gold NP in 2D array (geometry II) along principal symmetry directions calculated from MD simulations. $\mathrm{Q}^{*}$ is the reduced wavenumber. Strong phononic hybridization and emergence of kinks (Kohn anomaly) reflect the free standing particle phononic behavior.

Surface and free standing particle effects. In Fig.4 we show the behavior of terasonic excitations of a gold NP in $2 \mathrm{D}$ array, geometry $I I(\mathrm{x}=5 \mathrm{~nm}, \mathrm{y}=20 \mathrm{~nm})$. A striking difference with geometry I (see Fig.2) is that all transverse polarizations become double degenerate reflecting the isotropic nature of the liquid matrix. Gold NP array, being not as tightly-packed as in the previous case, exhibits resonant multiple scattering along all high symmetry directions. The strong intraband phononic hybridization emerges due to the finite size effect as a result of the expansion of the $2 \mathrm{D}$ gold NP array. In the present geometry, spherical symmetry and the surface effects of NPs become more pronounced, sharing characteristic phononic features with free-standing NPs. This interpretation is supported by recent exper-imental studies of low-frequency Raman spectroscopy. ${ }^{42}$ In fact, the computed dispersions exhibit phononic kinks and strong phononic hybridization in the $1.5 \sim 2.3 \mathrm{meV}$ energy range (see Fig.4). Remarkably, our MD calculations are in a good agreement with experimentally 

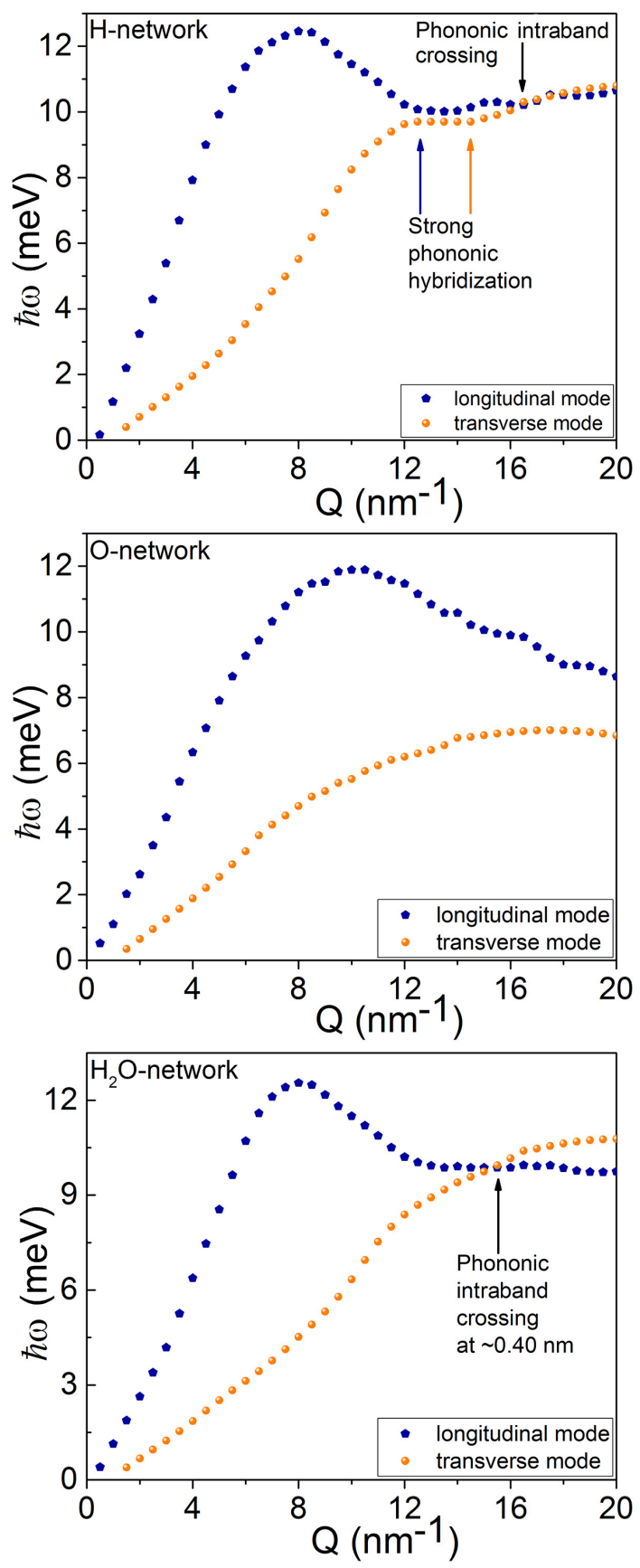

Figure 5: Dispersion relations are as in Fig.3, but referring to the geometry II. 
detected Raman peaks in the free standing gold NPs case, ${ }^{43}$ which were found at $19 \mathrm{~cm}^{-1}$ $(2.4 \mathrm{meV})$ and $11 \mathrm{~cm}^{-1}(1.4 \mathrm{meV})$ for Au NPs with a size of $3.2 \mathrm{~nm}$ and $4.8 \mathrm{~nm}$ respectively. The origin of these peaks has been assigned to the spheroidal $l=1, n=0$ normal vibration mode of the Au spherical surface. ${ }^{43}$ It is noteworthy that the phonon propagation in a NP array is strikingly different if the system is in vacuum or immersed in a liquid environment. When NPs are confined to a liquid matrix their phonon modes can interact through the medium which allows the vibrational energy of NPs to be transmitted to the liquid matrix; hence, the bound vibrational modes become resonant. ${ }^{8}$

To further strengthen the point that the phononic states in the water matrix are determined by the finite-size 2D gold NP geometry, we show the dispersion relations in Fig.5. Similarly to the previous geometry (see Fig.3), the phononic intraband excitations in Onetwork do not get mixed. This behavior stems from the different symmetry of the atomic networks: hexagonal for $\mathrm{H}$ atoms and tetrahedral for $\mathrm{O}$ ones. Furthermore, we notice that the water matrix becomes less sensitive to the presence of the $2 \mathrm{D}$ gold NP array upon approaching the free standing particle geometry. This can be readily inferred from the sound dispersions of $\mathrm{H}$ - and $\mathrm{H}_{2} \mathrm{O}$-networks which both exhibit Dirac-like crossing at about 15.7 $\mathrm{nm}^{-1}(\sim 0.4 \mathrm{~nm})$.

Dynamic and structural correlation functions of water. Molecules in the water matrix are very tightly packed, which implies that a water molecule in a liquid matrix is continuously interacting with its near neighbors. The forces exerted by these neighbors are sufficiently strong to hold the molecule rattling around in a confined pair correlated domain as long as the neighbors are forming a cage. However, due to the large nm-scale density fluctuations in the water matrix (see Fig.3 and Fig.5), such cage has a rather short life time (see Fig.6.d) and after a short time lapse the trapped molecule can escape from it. For our further analysis, in order to gain insight on the picosecond dynamics and nm-scale pair correlations of gold NPs in water matrix, we use the Green-Kubo formulas. In Fig.6.(a-c) we plot the partial pair distribution functions $g(r)$, while in Fig.6.d we depict the normalized velocity 
autocorrelation functions (VACF). The partial $g(r)$ shows how a reference particle, i.e. an atom or a molecule correlates with another one at a distance $r$, while the VACF measures how the dynamics of an individual atom or a molecule evolves in time. The obtained partial $g(r)$ are reported in Fig.6 as averaged over the course of a simulation run.
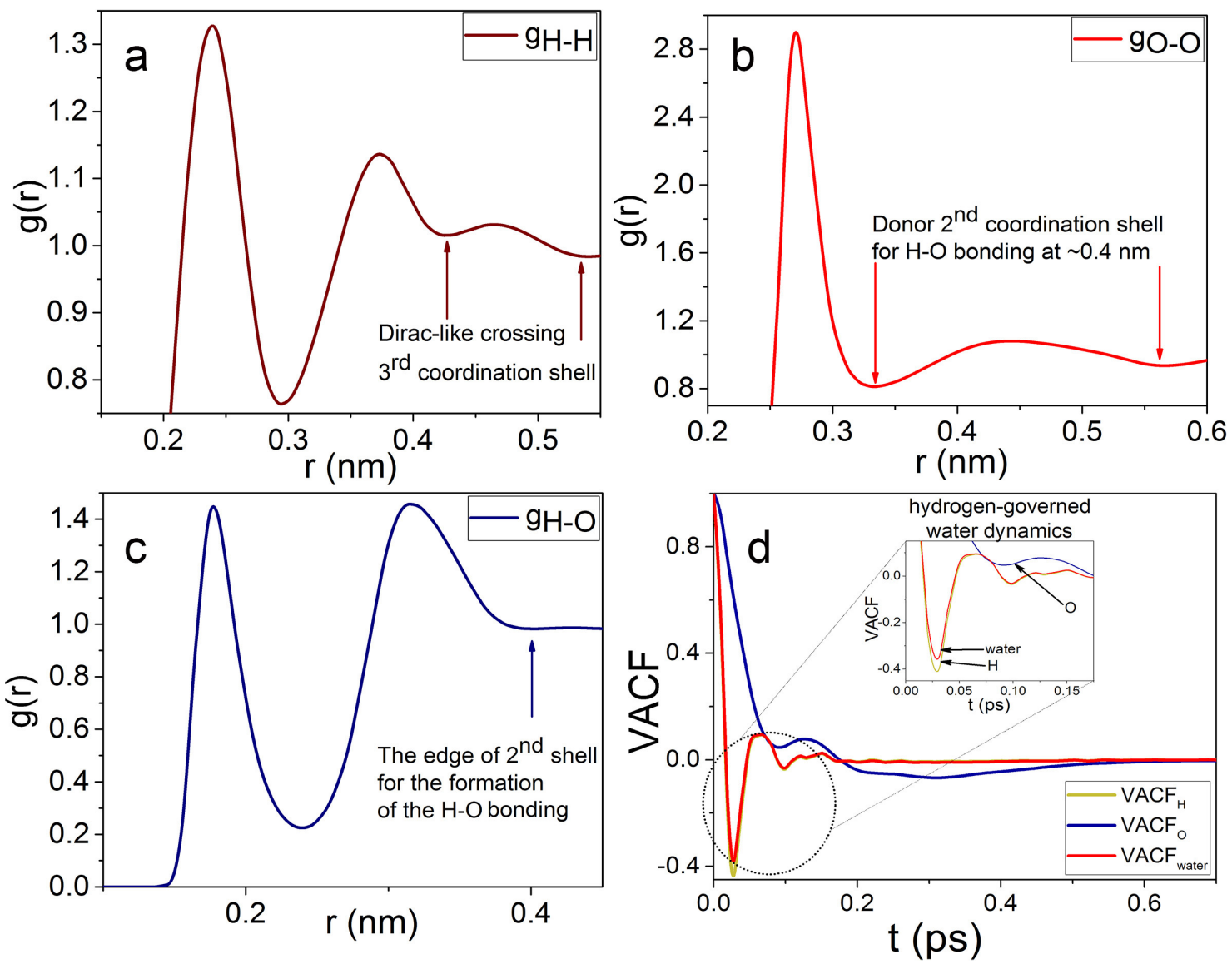

Figure 6: The boundary between pair correlated region of $\mathrm{H}-\mathrm{O}$ bond formation and disordered area. Pair correlations between hydrogen and oxygen atoms stick around for tiny fractions of a picosecond before decaying and are not supported beyond $\sim 0.4 \mathrm{~nm}$ at equilibrium. All partial $g(r)$ and VACF functions are the same for both geometries at the equilibrium.

In Fig.6.d (see inset) we provide an additional evidence that the dynamics of the water matrix is governed by hydrogen atoms as indicated by the proximity between the VACF of $\mathrm{H}_{2} \mathrm{O}$ and the one of $\mathrm{H}$. Thus, both phononic/collective excitations and single atom/ molecular dynamics of the water matrix are governed by H-network at nm-length and picosecond time scales. A Dirac-like phononic crossing (see Fig.3 and Fig.5) occurs at 0.41 $\mathrm{nm}$ and $0.40 \mathrm{~nm}$, 
respectively. These dynamic/phononic mixing happens due to the re-sticking of hydrogen and oxygen atoms from their $3^{\text {rd }}$ and $2^{\text {nd }}$ coordination shells, respectively. From Fig.6.c we observe that hydrogen bonds at the equilibrium have a boundary/edge between pair correlated or ordered $(g(r) \neq 1)$ and disordered structure $(g(r) \approx 1)$ at $\sim 0.4 \mathrm{~nm}$. Therefore, the distance $\mathrm{d}=0.4 \mathrm{~nm}$ can be identified as the average size of a pair correlated domain randomly oriented in space. We are thus urged to conclude that the finite size effect has also an impact on both the low- and high-frequency dynamics within a domain and beyond. The absence of pair correlations beyond a certain domain affects transverse low-frequency excitations only (see Fig.3 and Fig.5), since compression (longitudinal modes) sounds waves can easily propagate even in highly disordered media. These pair correlated local domains are isotropically dispersed in space and form a continuous media. Hence, boundaries be-tween these local domains lead to mixing of phononic polarizations. Therefore, ice-like pair correlated domains exhibit emergent-nucleation behavior at ps-time scale in the presence of spherical nano-defects and, as a result, they could be a precursor of ice-crystalline formation at ns-time scale. ${ }^{44}$

From Fig.6.d we see that oxygen molecules do not experience any collisions over the $\sim 0.2-$ 0.6 ps time lapse or $\sim 7-20 \mathrm{meV}$ energy range, which includes the Dirac-like crossing $(\sim 10$ meV). This suggests that a Dirac-like phononic mixing (Fig.3 and Fig.5) happens due to hydrogen-oxygen sub-picosecond re-sticking: either over rotational reconfigurations of $\mathrm{H}_{2} \mathrm{O}$ molecules or due to H-network sound bouncing off the domains' edges (echo effect). Likewise the arrangement of water molecules in ice-like aggregates is induced by the same mechanism as for H-governed phonon polarization mixing. For the geometry I, phononic crossing occurs at $0.41 \mathrm{~nm}$ (see Fig.3), which may correspond to a disordered form of the orthorhombic icelike structure (proton ordered phase). ${ }^{45}$ For the geometry $I I$, the phononic mixing takes place at $\sim 0.40 \mathrm{~nm}$ (see Fig.5), which may correspond to a disordered form of the tetragonal icelike structure (proton disordered phase). ${ }^{46}$ Each water molecule is H-bonded to four others, two as a donor and two as an acceptor. Tetragonal ice contains a screw-type H-bonded 
arrangement (right-handed double helix), ${ }^{47}$ thus, its Dirac-like THz phononic behavior can be an intrinsic effect in the presence of $2 \mathrm{D}$ gold NP arrays. Remarkably, orthorhombic ice structure is the proton-ordered form of tetragonal ice, and transition from one structure to another is known to occur via chemical doping. ${ }^{48}$ Here, we showed that such a transition can be triggered by structural engineering of 2D gold NP arrays too.

Overall, presented results provide a compelling evidence that one of the main effects induced in the water matrix structure by the tuning of the gold NP array geometry is the transition between a state with ordered hydrogen atoms (geometry $I$ ) to a state with disordered ones (geometry II). Interestingly, this is clearly represented by the transformation in the dispersion of the sound mode propagating through the H-H network (see Fig.3). For the geometry I, the more ordered H-network structure yields distinct transverse and longitudinal phononic branches (see Fig.3) across the whole explored Q-range. Interestingly, the dispersion curves are not only well separated (except for the Dirac-like crossing) but experience coherent Q-dephasing (the maximum of red dots coincides with a minimum of the blue ones, see Fig.3). Remarkably, for the geometry II, the H proton disorder causes transverse and longitudinal branches (see Fig.5) to converge essentially to the same frequency for $\mathrm{Q}^{\aleph} \geq 12 \mathrm{~nm}^{-1}$. This finding indicates that for the distance b eyond the threshold of $\mathrm{f}_{Q^{\aleph}}^{2 \pi}$ $\leq 0.52 \mathrm{~nm}$ longitudinal and transverse modes become physically indistinguishable, as the two orthogonal polarizations are strongly coupled with one another. This result is consistent with the evidence of a strong coupling between longitudinal and transverse phonon dynamics in bulk water at low temperature. ${ }^{49,50}$

Au correlations. The pair distribution function $g(r)$ (see Methods for detail) illustrates how the density of molecules changes with respect to distance. The calculated $g(r)$ (displayed in Fig.7) is in a good agreement with previous studies. ${ }^{51}$ It shows that Au-Au pair correlations at $\mathrm{r}=0.36 \mathrm{~nm}$ are almost absent, but $\mathrm{H}-\mathrm{O}$ pair correlations has a second peak located around $\mathrm{r}=0.36 \mathrm{~nm}$ (see Fig.6). It proves that the nature of the Dirac-like point in gold NPs (geometry I) along (110) high symmetry direction (see Fig.2) is a result of a confined water-induced 

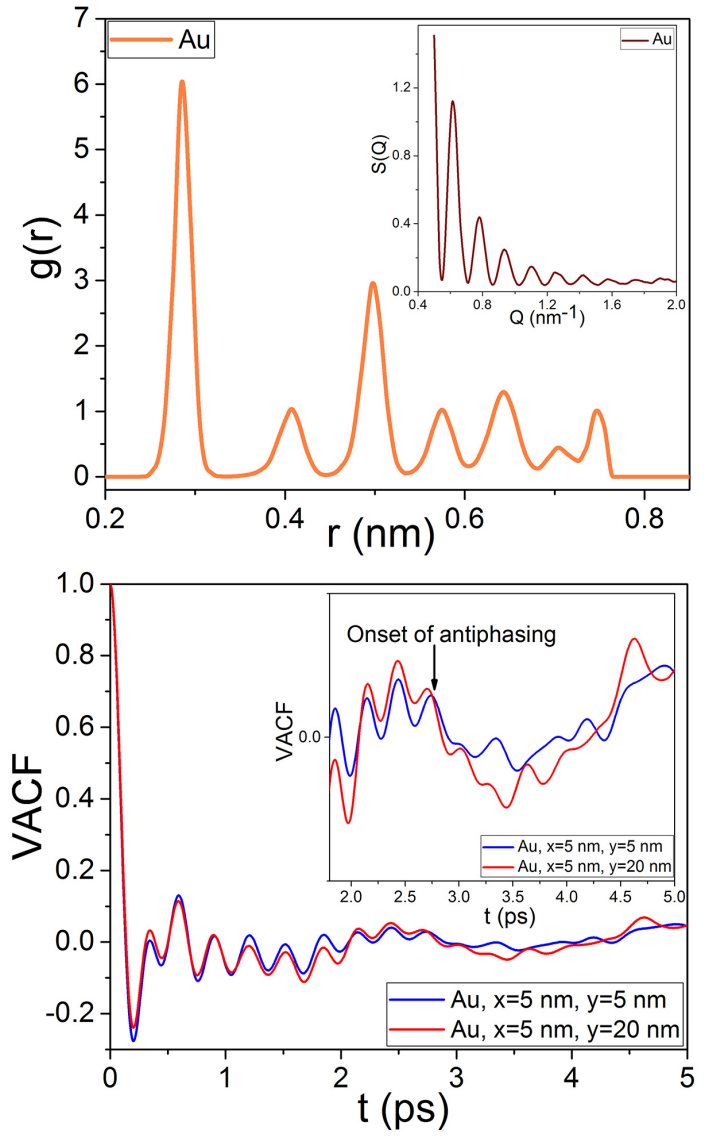

Figure 7: $g(r)$ and VACF of gold NP. 
effect. In Fig.7 we also demonstrate velocity autocorrelation function for both geometries. It is noteworthy that the onset of out of phasing of the Au particles dynamics (see Fig.7) emerges at $\sim 2.5$ ps-time scale. The origin of this dephasing is assigned to the electron-electron thermalization $(0.4 \sim 2 \mathrm{ps})$ followed by the electron-phonon relaxation processes $(2 \sim 5 \mathrm{ps}) .{ }^{52}$ Both processes are the result of a non-Fermi behavior (a Fermi behavior occurs at the 10 fs time scale) of the conduction electrons in gold NPs when electron-surface scattering is the dominant relaxation process. ${ }^{52}$ Because of the dephasing ${ }^{53}$ (2.5 ps or equivalently $\left.1.65 \mathrm{meV}\right)$ in $\mathrm{Au}$ dynamics being originated from the surface electron-phonon relaxation processes, ${ }^{54}$ it significantly enhances the resonant multiple phononic scattering leading to the Kohn anomaly ${ }^{35}$ in phonon dispersions (see Fig.4).

Conclusion. Sound control and manipulation in soft matter systems at THz scale is not well-studied yet and it needs to be thoroughly explored, considering the large variety of potential applications. The interplay of light, sound and heat at nanoscale reveals a realm, which brings new challenges at the boundaries between physics, chemistry, and nanotechnology. As a meaningful example of the intriguing phononic behavior of hybrid systems, we have here considered 2D gold NP arrays in a water matrix. A systematic computer simulation study on this system has enabled us to observe surprising effects originated by the interplay between $\mathrm{THz}$ density fluctuations and the nanoscale structural arrangements. Specifically, we found that phonon propagation through $\mathrm{H}\left(\mathrm{H}_{2} \mathrm{O}\right)$ atomic (molecular) network bears ev-idence for the Dirac-like intraband crossings of phononic branches. Whereas, the phononic excitations in Au NP's network is significantly influenced by the presence of the water ma-trix and strongly dependent on the geometry of the NP's arrangement. In particular, this triggers the onset of Kohn anomalies in the longitudinal modes of the Au NP's networks as well as the two-fold degeneracy of the transverse ones (surface and free standing particle effects), which reflects the isotropic nature of the liquid matrix.

We also observe that phonon-like excitations propagating over the network of $\mathrm{H}\left(\mathrm{H}_{2} \mathrm{O}\right)$ atoms (molecules) can cause polarizations to mix upon reaching the boundary of locally 
ordered (pair correlated) domains. Remarkably, non-trivial effects were observed not only in the dynamic response but also in the local structural arrangement of the water matrix. In fact, the confinement in the interstices between neighboring gold NPs (lattice and finite size effects) induces a re-structuring of water molecules resulting in hexagonal-like local aggregates. Thus, it can be considered as a phononic fingerprint of emergent nucleation of nm-sized ice-like structures at ps time scale.

Finally, we found that by varying the geometry of gold NP arrays the sub-terahertz phononics in a soft matter system can be probed. We showed that by expanding the geometry $I$ in $y$-direction (asymmetric expanding), both collective and single particle dynamics of $\mathrm{Au}$ atoms change. For the former it leads to the strong intraband phononic hybridization (Kohn anomaly) consistent with previous Raman measurements. For the latter it causes the dephasing in NP's dynamics on ps-time scale which was also observed in ultrafast laser spectroscopy experiments. Therefore, the phononic excitations in gold NP arrays can be suitably tuned via structural engineering. As a useful application, such light-absorbing Au NPs arrays in a water matrix could be used to enhance the efficiency of processes involving distillation. Another meaningful application could be sound control at $\mathrm{THz}$ scale. A manipulation of a focused sound wave in $\mathrm{THz}$ frequency with a nanometer precision via structural engineering may greatly advance novel ultrasound therapeutic technologies towards the treatment of oncology-related diseases and advancement of ultrasound-mediated drug delivery techniques.

Methods. Molecular dynamics simulations. We employed MD simulations in the well-known large-scale atomic/molecular massively-parallel dynamic simulator "LAMMPS". ${ }^{55}$ The computational unit cell contains 1260101 water molecules and $1979 \times n$ Au atoms where $n=6$ is the number of gold NPs. These numbers have been chosen so that the $2 \mathrm{D}$ gold NP array is commensurate with the water matrix (size: $48.4 \mathrm{~nm}, 91.4 \mathrm{~nm}, 8.6 \mathrm{~nm}$ ) when performing simulations with periodic boundaries. There are no directional constraints on vibrational modes in the system. Gold nanoparticles have the fcc structure with the lattice parameter $a=0.408 \mathrm{~nm}$. The embedded atom method (EAM) is widely used to describe the 
interaction between metallic atoms. In this paper, for $\mathrm{Au}-\mathrm{Au}$ modeling, the (EAM) potential $^{56}$ was used. The orientation of $\mathrm{Au}$ crystalites in $2 \mathrm{D}$ arrays is equivalent. Interactions among water molecule's components were modeled via the standard force field TIP3P. The interactions between $\mathrm{H}_{2} \mathrm{O}$ molecules and $\mathrm{Au}$ atoms were computed using the potential with a cutoff of $1.0 \mathrm{~nm} .{ }^{36}$ The constant pressure and temperature NPT ensemble was employed with a pressure of 1 atm controlled using Nose-Hoover barostat. A typical MD simulation lasted about 200 ps, and the properties were averaged over the last 100 ps of simulation, preceded by 100 ps of equilibration. A detailed MD simulation procedure of $2 \mathrm{D}$ gold NP arrays in the water matrix is analogous to a recent work. ${ }^{57}$

Transverse and longitudinal correlation functions. Longitudinal and transverse currents can be represented as

$$
\begin{aligned}
J_{L}(\mathbf{Q}, t) & =\sum_{i=1}^{N} \hat{\mathbf{Q}}_{\|} \cdot \mathbf{v}_{i}(t) \exp \left[-i \mathbf{Q} \cdot \mathbf{r}_{i}(t)\right] \\
J_{T}(\mathbf{Q}, t) & =\sum_{i=1}^{N} \hat{\mathbf{Q}}_{\perp} \cdot \mathbf{v}_{i}(t) \exp \left[-i \mathbf{Q} \cdot \mathbf{r}_{i}(t)\right]
\end{aligned}
$$

where $\hat{\mathbf{Q}}_{\|}$and $\hat{\mathbf{Q}}_{\perp}$ are unit vectors parallel and perpendicular to $\mathbf{Q}$, respectively. $\mathbf{r}_{i}(t)$ and $\mathbf{v}_{i}(t)$ denote the atoms' position and velocity, respectively. The frequency decomposition of the current autocorrelation functions can be determined as

$$
C_{\alpha}(Q, \omega) \equiv \int<J_{\alpha}(Q, t) J_{\alpha}(-Q, 0)>e^{i \omega t} d t
$$

where $\alpha=(L, T)$ and $<>$ represents the average over initial conditions. Phonon dispersion relations (see Figs.2-5) were derived from the position of the dominant peaks of the $C_{\alpha}(Q, \omega)$ function.

Pair distribution function. The $g(r)$ (see Figs.6-7) can be represented via the static 
structure factor $S(Q)$

$$
g(r)=1+\frac{1}{2 \pi \varrho} \int_{0}^{\infty}[S(Q)-1] \frac{\sin Q r}{Q r} Q^{2} d Q
$$

which can be measured in X-ray diffraction experiments. $S(q)$ is the static structure factor and $\varrho=\frac{N}{V}$ is the number density. ${ }^{10}$

Velocity autocorrelation function. The normalized VACF (see Figs.6-7) can be written as

$$
C(t)=\frac{\langle\mathbf{v}(0) \cdot \mathbf{v}(t)>}{\langle\mathbf{v}(0) \cdot \mathbf{v}(0)>}
$$

where $\mathbf{v}(t)$ is the velocity vector of an atomic system and $<>$ denotes the average over initial conditions. ${ }^{10}$

\section{Acknowledgement}

We thank Oleg Gang, Ron Pindak, Yugang Zhang and Alexander Balatsky for fruitful discussions. This research used resources of the National Synchrotron Light Source II, a U.S. Department of Energy (DOE) Office of Science User Facility operated for the DOE Office of Science by Brookhaven National Laboratory under Contract No. DE-SC0012704.

\section{References}

(1) Maldovan, M. Sound and heat revolutions in phononics. Nature 2013, 503, 209-217.

(2) Cheng, W.; Gomopoulos, N.; Fytas, G.; Gorishnyy, T.; Walish, J.; Thomas, E. L.; Hiltner, A.; Baer, E. Phonon Dispersion and Nanomechanical Properties of Periodic 1D Multilayer Polymer Films. Nano Lett. 2008, 8(5), 1423-1428.

(3) Still, T.; Cheng, W.; Retsch, M.; Sainidou, R.; Wang, J.; Jonas, U.; Stefanou, N.; Fytas, G. Simultaneous Occurrence of Structure-Directed and Particle-Resonance-Induced Phononic Gaps in Colloidal Films. Phys. Rev. Lett. 2008, 100, 194301. 
(4) Yang, L.; Yang, N.; Li, B. Extreme Low Thermal Conductivity in Nanoscale 3D Si Phononic Crystal with Spherical Pores. Nano Lett. 2014, 14(4), 1734-1738.

(5) Hossein Mousavi, S.; Khanikaev, B.; Wang, Z. A new class of tunable hypersonic phononic crystals based on polymer-tethered colloids. Nat. Commun. 2015, 6, 8309.

(6) Lee, J.; Lim, J.; Yang, P. Ballistic Phonon Transport in Holey Silicon. Nano Lett. 2015, 15(5), 3273-3279.

(7) Gomopoulos, N.; Maschke, D.; Koh, C. Y.; Thomas, E. L.; Tremel, W.; Butt, H. J.; Fytas, G. One-Dimensional Hypersonic Phononic Crystals. Nano Lett. 2010, 10(3), 980-984.

(8) Still, T.; Gantzounis, G.; Kiefer, D.; Hellmann, G.; Sainidou, R.; Fytas, G.; Stefanou, N. Collective Hypersonic Excitations in Strongly Multiple Scattering Colloids. Phys. Rev. Lett. 2011, 106, 175505.

(9) Alonso-Redondo, E.; Schmitt, M.; Urbach, Z.; Hui, C. M.; Sainidou, R.; Rembert, P.; Matyjaszewski, K.; Bockstaller, M. R.; Fytas, G. A new class of tunable hypersonic phononic crystals based on polymer-tethered colloids. Nat. Commun. 2015, 6, 8682.

(10) Bolmatov, D.; Zav'yalov, D.; Zhernenkov, M.; Musaev, E. T.; Cai, Y. Q. Unified phonon-based approach to the thermodynamics of solid, liquid and gas states. Ann. Phys. 2015, 363, 221-242.

(11) Bolmatov, D.; Zhernenkov, M.; Zav'yalov, D.; Stoupin, S.; Cunsolo, A.; Cai, Y. Q. Thermally triggered phononic gaps in liquids at THz scale. Sci. Rep. 2016, 6, 19469.

(12) Bolmatov, D.; Zhernenkov, M.; Zav'yalov, D.; Stoupin, S.; Cai, Y. Q.; Cunsolo, A. Revealing the Mechanism of the Viscous-to-Elastic Crossover in Liquids. J. Phys. Chem. Lett. 2015, 6(15), 3048-3053. 
(13) Bolmatov, D.; Musaev, E. T.; Trachenko, K. Symmetry breaking gives rise to energy spectra of three states of matter. Sci. Rep. 2013, 3, 2794.

(14) Zhernenkov, M.; Bolmatov, D.; Soloviov, D.; Zhernenkov, K.; Toperverg, B. P.; Cunsolo, A.; Bosak, A.; Cai, Y. Q. Revealing the mechanism of passive transport in lipid bilayers via phonon-mediated nanometre-scale density fluctuations. Nat. Commun. 2016, 7, 11575 .

(15) Li, M.; Chu, X. Q.; Fratini, E.; Baglioni, P.; Alatas, A.; Alp, E. E.; Chen, S. H. Phononlike excitation in secondary and tertiary structure of hydrated protein powders. Soft Matter 2011, 7(21), 9848-9853.

(16) Cunsolo, A.; Suvorov, A.; Cai, Y. Q. The onset of shear modes in the high frequency spectrum of simple disordered systems: current knowledge and perspectives. Philos. Mag. 2016, 96, 732-742.

(17) Zhu, J.; Zhang, H.; Kotov, N. A. Thermodynamic and Structural Insights into Nanocomposites Engineering by Comparing Two Materials Assembly Techniques for Graphene. ACS Nano 2013, 7(6), 4818-4829.

(18) Cha, S. H.; Hong, J.; McGuffie, M.; Yeom, B.; VanEpps, J. S.; Kotov, N. A. ShapeDependent Biomimetic Inhibition of Enzyme by Nanoparticles and Their Antibacterial Activity. ACS Nano 2015, 9(9), 9097-9105.

(19) Zhao, Y.; Xu, L.; Ma, W.; Wang, L.; Kuang, H.; Xu, C.; Kotov, N. A. Shell-Engineered Chiroplasmonic Assemblies of Nanoparticles for Zeptomolar DNA Detection. Nano Lett. 2014, $14(7), 3908-3913$.

(20) Tian, Y.; Wang, T.; Liu, W.; Xin, H. L.; Li, H.; Ke, Y.; Shih, W. M.; Gang, O. Prescribed nanoparticle cluster architectures and low-dimensional arrays built using octahedral DNA origami frames. Nat. Nanotechnol. 2015, 10, 637-644. 
(21) Lu, F.; Yager, K. G.; Zhang, Y.; Xin, H.; Gang, O. Superlattices assembled through shape-induced directional binding. Nat. Commun. 2015, 6, 6912.

(22) Miller, D.; Smith, N.; Bailey, M.; Czarnota, G.; Hynynen, K.; Makin, I. Overview of Therapeutic Ultrasound Applications and Safety Considerations. J. Ultrasound Med. 2012, 31(4), 623-634.

(23) Lukianova-Hleb, X., E. Y. Ren; Sawant, R. R.; Wu, X.; Torchilin, V. P.; Lapotko, D. O. On-demand intracellular amplification of chemoradiation with cancer-specific plasmonic nanobubbles. Nat. Med. 2014, 20, 778-784.

(24) Rechtsman, M. C.; Zeuner, J. M.; Plotnik, Y.; Lumer, Y.; Podolsky, D.; Dreisow, F.; Nolte, S.; Segev, M.; Szameit, A. Photonic Floquet topological insulators. Nature 2013, 496, 196-200.

(25) Kariyado, T.; Hatsugai, Y. Manipulation of Dirac Cones in Mechanical Graphene. Sci. Rep. 2015, 5, 18107.

(26) Wang, P.; Lu, L.; Bertoldi, K. Topological Phononic Crystals with One-Way Elastic Edge Waves. Phys. Rev. Lett. 2015, 115, 104302.

(27) Zhang, X. Observing Zitterbewegung for Photons near the Dirac Point of a TwoDimensional Photonic Crystal. Phys. Rev. Lett. 2008, 100, 113903.

(28) Weick, G.; Woollacott, C.; Barnes, W. L.; Hess, O.; Mariani, E. Dirac-like Plasmons in Honeycomb Lattices of Metallic Nanoparticles. Phys. Rev. Lett. 2013, 110, 106801.

(29) Kane, C. L.; Lubensky, T. C. Topological boundary modes in isostatic lattice. Nat. Phys. 2014, 10, 39-45.

(30) Zhang, L.; Niu, Q. Chiral Phonons at High-Symmetry Points in Monolayer Hexagonal Lattices. Phys. Rev. Lett. 2015, 115, 115502. 
(31) Mousavi, S. H.; Khanikaev, A. B.; Wang, Z. Topologically protected elastic waves in phononic metamaterials. Nat. Commun. 2015, 6, 8682.

(32) Süsstrunk, R.; Huber, S. D. Observation of phononic helical edge states in a mechanical topological insulator. Science 2015, 349, 47-50.

(33) Sauceda, H. E.; Salazar, F.; Pérez, L. A.; Garzón, I. L. Size and Shape Dependence of the Vibrational Spectrum and Low-Temperature Specific Heat of Au Nanoparticles. J. Phys. Chem. C 2013, 117(47), 25160-25168.

(34) Moriarty, J. A. Total Energy of Copper, Silver, and Gold. Phys. Rev. B 1972, 6, 1239.

(35) Hoesch, M.; Bosak, A.; Chernyshov, D.; Berger, H.; Krisch, M. Giant Kohn Anomaly and the Phase Transition in Charge Density Wave ZrTe3. Phys. Rev. Lett. 2009, 102, 086402 .

(36) Merabia, S.; Shenogin, S.; Joly, L.; Keblinski, P.; Barrat, J. L. Heat transfer from nanoparticles: A corresponding state analysis. Proc. Natl. Acad. Sci. U.S.A. 2009, $106,15113-15118$.

(37) Śmiechowski, M.; Schran, C.; Forbert, H.; Marx, D. Correlated Particle Motion and THz Spectral Response of Supercritical Water. Phys. Rev. Lett. 2016, 116, 027801.

(38) Richardson, J. O.; Pérez, C.; Lobsiger, S.; Reid, A. A.; Temelso, B.; Shields, G. C.; Kisiel, Z.; Wales, D. J.; Pate, B. H.; Althorpe, S. C. Concerted hydrogen-bond breaking by quantum tunneling in the water hexamer prism. Science 2016, 351, 1310-1313.

(39) Gorshunov, B. P.; Zhukova, E. S.; Torgashev, V. I.; Lebedev, V. V.; Shakurov, G. S.; Kremer, R. K.; Pestrjakov, E. V.; Thomas, V. G.; Fursenko, D. A.; Dressel, M. Quantum Behavior of Water Molecules Confined to Nanocavities in Gemstones. J. Phys. Chem. Lett. 2013, 4(12), 2015-2020. 
(40) Kolesnikov, A. I.; Reiter, G. F.; Choudhury, N.; Prisk, T. R.; Mamontov, E.; Podlesnyak, A.; Ehlers, G.; Seel, A. G.; Wesolowski, D. J.; Anovitz, L. M. Quantum Tunneling of Water in Beryl: A New State of the Water Molecule. Phys. Rev. Lett. 2016, 116, 167802 .

(41) Mosaddeghi, H.; Alavi, S.; Kowsari, M. H.; Najafi, B. Simulations of structural and dynamic anisotropy in nano-confined water between parallel graphite plates. J. Chem. Phys. 2012, 137, 184703.

(42) Yadav, H. K.; Gupta, V.; Sreenivas, K.; Singh, S. P.; Sundarakannan, B.; Katiyar, R. S. Low Frequency Raman Scattering from Acoustic Phonons Confined in ZnO Nanoparticles. Phys. Rev. Lett. 2006, 97, 085502.

(43) Mankad, V.; Jha, P. K.; Ravindran, T. R. Probing confined acoustic phonons in free standing small gold nanoparticles. J. Appl. Phys. 2013, 113, 074303.

(44) English, N. J.; Tse, J. S. Massively parallel molecular dynamics simulation of formation of ice-crystallite precursors in supercooled water: Incipient-nucleation behavior and role of system size. Phys. Rev. E 2015, 92, 032132.

(45) Salzmann, C. G.; Radaelli, P. G.; Hallbrucker, A.; Mayer, E.; Finney, J. L. The preparation and structure of hydrogen ordered phases of ice. Science 2006, 311, 1758-1761.

(46) Koza, M.; Schober, H.; Tlle, A.; Fujara, F.; Hansen, T. Formation of ice XII at different conditions. Nature 1999, 397, 660-661.

(47) O'Keeffe, M. New ice outdoes related nets in smallest-ring size. Nature 1998, 392, 879.

(48) Salzmann, C. G.; Hallbrucker, A.; Finney, J. L.; Mayer, E. Raman spectroscopic features of hydrogen-ordering in ice XII. Chem. Phys. Lett. 2006, 429, 469-473.

(49) Sampoli, M.; Ruocco, G.; Sette, F. Mixing of Longitudinal and Transverse Dynamics in Liquid Water. Phys. Rev. Lett. 1997, 79, 1678. 
(50) Pontecorvo, E.; Krisch, M.; Cunsolo, A.; Monaco, G.; Mermet, A.; Verbeni, R.; Sette, F.; Ruocco, G. High-frequency longitudinal and transverse dynamics in water. Phys. Rev. E 2005, 71, 011501.

(51) Chui, Y. H.; Snook, I. K.; Russo, S. P. Visualization and analysis of structural ordering during crystallization of a gold nanoparticle. Phys. Rev. B 2007, 76, 195427.

(52) Link, S.; Burda, C.; Wang, Z. L.; El-Sayed, M. A. Electron dynamics in gold and goldsilver alloy nanoparticles: The influence of a nonequilibrium electron distribution and the size dependence of the electron-phonon relaxation. J. Chem. Phys. 1999, 111, 1255.

(53) Hodak, J. H.; Henglein, A.; Hartland, G. V. Size dependent properties of Au particles: Coherent excitation and dephasing of acoustic vibrational modes. J. Chem. Phys. 1999, $111,8613$.

(54) Burda, C.; Chen, X.; Narayanan, R.; El-Sayed, M. A. Chemistry and Properties of Nanocrystals of Different Shapes. Chem. Rev. 2005, 105(4), 1025-1102.

(55) Plimpton, S. Fast Parallel Algorithms for Short-Range Molecular Dynamics. J. Comput. Phys. 1995, 117, 1-19.

(56) Foiles, S. M.; Baskes, M. I.; Daw, M. S. Embedded-atom-method functions for the fcc metals $\mathrm{Cu}, \mathrm{Ag}, \mathrm{Au}, \mathrm{Ni}, \mathrm{Pd}, \mathrm{Pt}$, and their alloys. Phys. Rev. B 1986, 33, 7983.

(57) Chen, X.; Munjiza, A.; Zhang, K.; Wen, D. Molecular Dynamics Simulation of Heat Transfer from a Gold Nanoparticle to a Water Pool. J. Phys. Chem. C 2014, 118, $1285-1293$. 


\section{Graphical TOC Entry}
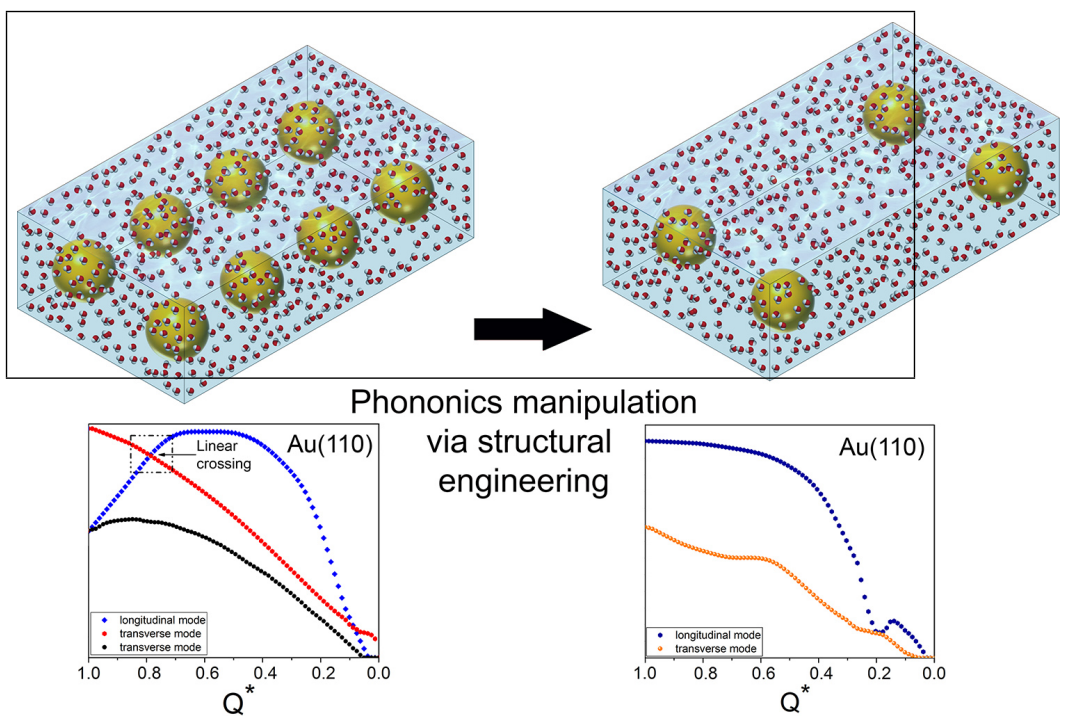